\title{
IMPACTOS DOS ANOS CLIMÁTICOS EXTREMOS NO RENDIMENTO DA LAVOURA TEMPORÁRIA DE MANDIOCA NA REGIÃO RURAL DA METRÓPOLE DE BELÉM - PARÁ
}

\author{
SOUZA, Paulo Fernando de Souza - pfsouza@ufpa.br \\ Universidade Federal do Pará/ UFPA
} SOUZA, Everaldo Barreiros de - everaldo@ufpa.br
Instituto Tecnológico Vale/ITV

QUEIROZ, Joaquim Carlos Barbosa - joaquim@ufpa.br Universidade Federal do Pará/ UFPA

\section{SILVA JUNIOR, João de Athaydes - athaydes@ufpa.br} Universidade Federal do Pará/ UFPA

\begin{abstract}
RESUMO: Este trabalho apresenta uma contribuição aos estudos sobre os impactos do clima regional na agricultura da Amazônia oriental, particularmente da lavoura temporária de mandioca (Manihot esculenta Crantz). Foram utilizados dados da base municipal da Região Rural da Metrópole de Belém (RRB) no estado do Pará com informações agrícolas (área colhida, quantidade produzida e rendimento médio) e climatológicas (precipitação) no período de 1990 a 2014. Os resultados das correlações evidenciaram que o regime pluviométrico anual afeta o rendimento da mandioca em diversos municípios, sendo que identificou-se um padrão heterogêneo com valores positivos e negativos se alternando ao longo da região. As análises de impactos nos anos climáticos extremos EC- (El Niño no Pacífico e GrNorte no Atlântico) e EC+ (La Niña no Pacífico e GrSul no Atlântico) mostraram uma resposta diferente ao longo da região com sinais favoráveis e desfavoráveis ao rendimento médio de mandioca.
\end{abstract}

Palavras- chaves: Climatologia Agrícola, Amazônia, Impactos regionais.

IMPACTS OF EXTREME CLIMATE YEARS ON CASSAVA FARMING IN THE RURAL REGION OF METROPOLIS OF BELÉM - STATE OF PARÁ

\begin{abstract}
This paper presents a contribution to the studies on the impacts of the regional climate on agriculture in eastern Amazon, particularly on the temporary farming of cassava (Manihot esculenta Crantz). Data from the municipal base of the Rural Region of Metropolis of Belém (RRB) in the state of Pará were used for agricultural (crop area, quantity produced and yield) and climatological (precipitation) informations from 1990 to 2014. The results of the correlations showed that the annual pluviometric regime affects cassava yield in several municipalities, so that a heterogeneous pattern with positive and negative values are identified throughout the region. Impacts analyses for extreme climatic years EC- (EI Niño in the Pacific and GrNorte in the Atlantic) and EC+ (La Niña in the Pacific and GrSul in the Atlantic) showed a different response along the region with favorable and unfavorable signs of the cassava yield.
\end{abstract}

Keywords: Agricultural Climatology, Amazon, Regional impacts

\section{INTRODUÇÃO}

A mandioca (Manihot esculenta Crantz) é considerada uma das maiores heranças da civilização indígena, com seu cultivo iniciado há 3.500 anos, possivelmente domesticada pelos tupis na Bacia Amazônica (ROOSEVELT et al., 1996). Atualmente, a mandioca é a quarta cultura de produção de alimentos mais importante do mundo, segundo dados da Organização das Nações Unidas para a Agricultura e Alimentação (FAO,2014), principalmente na região tropical, pois sua raiz e demais subprodutos são consumidos por mais de 800 milhões de pessoas. 
A cultura da mandioca é largamente cultivada em todo o território brasileiro, de norte a sul, em virtude de ser uma cultura explorada, em sua maioria, pelo segmento de pequenos produtores. A região Norte é a maior produtora de mandioca do Brasil, em termos quantitativos (produção em toneladas), superando a produção da região Nordeste, que desde 1990 era a maior produtora, tanto em área plantada quanto em quantidade produzida (MODESTO JUNIOR, ALVES, SILVA, 2016). O estado do Pará destaca-se como o principal estado produtor de mandioca do Brasil. Ao longo de vários anos o estado assume o topo em relação ao volume produzido em toneladas.

No contexto da meteorologia ou climatologia agrícola, de acordo com Ayoade (1986), as práticas agrícolas ocorrem a céu aberto, portanto, dependem diretamente do comportamento dos elementos climáticos, tal que a ocorrência de anomalias ou extremos meteorológicos resulta em impactos que afetam a produtividade e o manejo das culturas. Assim, a variabilidade pluviométrica nas regiões tropicais, principalmente para as culturas temporárias, como é o caso da mandioca, é a que mais condiciona os aspectos do plantio e rendimento agrícola, pois assume papel de fornecer o aporte hídrico para o período fenológico das culturas. Neste contexto, a precipitação é considerada um elemento climático fundamental no ciclo produtivo das plantas e, por conseguinte, na produção agrícola. Portanto, o conhecimento aprofundado sobre os padrões espaciais e temporais de precipitação pluviométrica é primordial nas atividades do setor produtivo, a exemplo da Agricultura, que no Brasil possui importância de dimensões social, econômica e ambiental (ARRUDA e PINTO, 1980; MANOSSO, 2005; FERREIRA, 2005).

Segundo os trabalhos de Dias e Martinez (1986) e Normanha (1941), a precipitação pluviométrica é um dos fatores de maior importância para o cultivo da mandioca, por tratar-se de lavoura cultivada quase que exclusivamente sem irrigação. O regime de chuvas, considerado mais adequado, é a ocorrência de um total anual de 1.000 a $1.500 \mathrm{~mm}$, com boa distribuição durante 6 a 8 meses do ciclo vegetativo. A deficiência de água nos primeiros cinco meses de cultivo da mandioca além de provocar grandes reduções na produção de raízes, pode causar até a morte das plantas. Para a região da Amazônia há poucos estudos com a abordagem de clima e agricultura. Teracines (2000) estudou os impactos econômicos do El Niño 97/98 na produção da mandioca na região norte que sofreu perda nesse período, passando de 5.033.699 ton em 1996/97 para 4.392.828 em 1997/1998, ou seja, ocorreu uma variação negativa de 12,7\% na produção.

No presente trabalho apresenta-se uma contribuição à climatologia agrícola da Amazônia oriental, particularmente para a Região Rural da Metrópole de Belém (RRB), abrangendo grande parte do norte e nordeste do estado do Pará, durante o período de 1990 a 2014. Investigam-se as relações entre o regime pluviométrico anual e o rendimento da mandioca (RM) nos municípios da RRB, bem como documentam-se os impactos regionais dos anos climáticos extremos na produtividade da mandioca.

\section{MATERIAL E MÉTODOS}

\section{ÁREA DE ESTUDO E DADOS USADOS}


Adotou-se como área de estudo a Região Rural da Metrópole de Belém (RRB), a qual foi definida pelo IBGE (2015) através do Projeto Regiões Rurais ${ }^{1}$ que concebeu uma nova divisão regional do Brasil a partir da dinâmica geográfica traçada pela produção agroindustrial no território nacional.

A Tabela 1 enumera os códigos e nomes dos municípios e a Figura 1 ilustra o mapa da área de estudo com a localização e domínio geográfico de um total de 78 municípios pertencentes à RRB. Observa-se a existência de uma extensa rede de drenagem hidrológica (Fig. 1, linhas em azul) na região, sendo que, dentre os rios principais, citam-se: o alto curso do rio Amazonas na parte oeste da região, nos municípios de Gurupá (30), Breves (15) e Afuá (3); o alto curso do rio Tocantins na porção centro-sul da região, nos municípios de Baião (9), Mocajuba (43) e Cametá (19), e os rios Guamá, Acará e Capim que atingem grande parte dos municípios situados nas porções leste e nordeste da RRB. Exceto nas áreas a oeste (principalmente na ilha de Marajó), verifica-se uma ampla cobertura de rodovias (Fig. 1, linhas em vermelho no mapa menor à direita) interligando os municípios ao longo da RRB

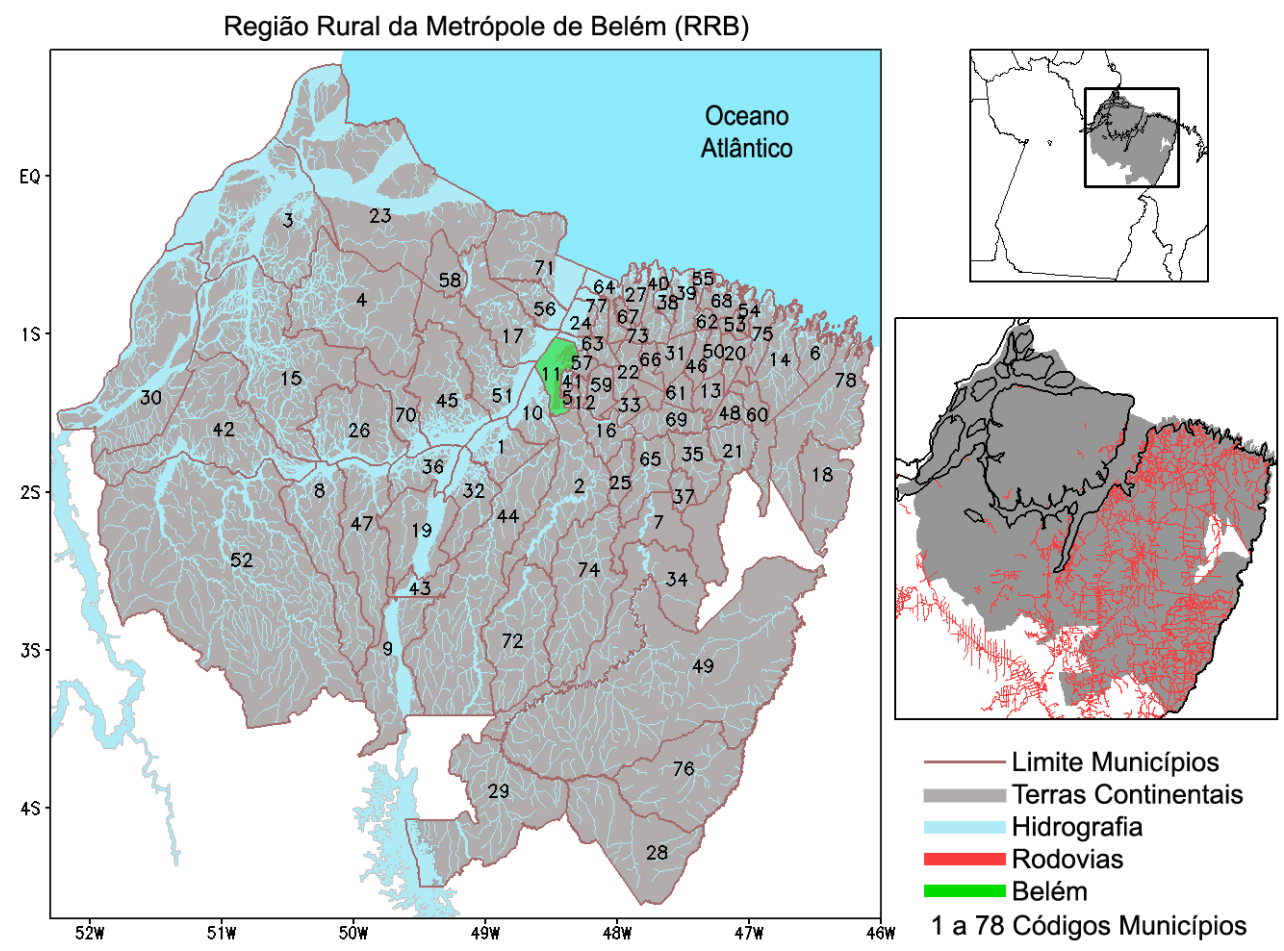

Figura 1 - Mapa da área de estudo com a localização e domínio geográfico dos municípios pertencentes à Região Rural da Metrópole de Belém (RRB). Em verde destacase o município de Belém e os números de 1 a 78 indicam os municípios segundo os códigos e nomes listados na Tabela 1. Fonte: Elaborado pelo autor.

\footnotetext{
${ }^{1}$ Neste mapeamento e regionalização do espaço rural Brasileiro foram usadas as bases conceituais desenvolvidas por Veltz (1999) e Haesbaert (1999) sobre território-rede e território-zona. Concernente ao território-rede, o critério definidor é o grau de centralidade e comando que as cidades e suas funções exercem sobre o campo, sendo importante a mensuração dos diversos fluxos e redes intra e inter-regionais. Quanto ao território-zona ou de uso contínuo, entende-se pelos usos e atividades dominantes do espaço rural, incluindo também as grandes extensões do território delimitadas por legislação especial, como é o caso das Terras Indígenas e Unidades de Conservação, as quais possuem importância particular na Amazônia.
} 
Tabela 1 - Listagem dos códigos e nomes dos municípios da Região Rural da Metrópole de Belém (RRB) no estado do Pará.

\begin{tabular}{cl|cl|cl}
\hline Código \# & Nome município & Código \# & Nome município & Código \# & Nome município \\
\hline $\mathbf{1}$ & Abaetetuba & $\mathbf{2 7}$ & Curuçá & $\mathbf{5 3}$ & Primavera \\
$\mathbf{2}$ & Acará & $\mathbf{2 8}$ & Dom Eliseu & $\mathbf{5 4}$ & Quatipuru \\
$\mathbf{3}$ & Afuá & $\mathbf{2 9}$ & Goianésia Pará & $\mathbf{5 5}$ & Salinópolis \\
$\mathbf{4}$ & Anajás & $\mathbf{3 0}$ & Gurupá & $\mathbf{5 6}$ & Salvaterra \\
$\mathbf{5}$ & Ananindeua & $\mathbf{3 1}$ & Igarapé-Açu & $\mathbf{5 7}$ & S Bárbara do Pará \\
$\mathbf{6}$ & Augusto Corrêa & $\mathbf{3 2}$ & Igarapé-Miri & $\mathbf{5 8}$ & Santa Cruz do Arari \\
$\mathbf{7}$ & Aurora do Pará & $\mathbf{3 3}$ & Inhangapi & $\mathbf{5 9}$ & Santa Isabel do Pará \\
$\mathbf{8}$ & Bagre & $\mathbf{3 4}$ & Ipixuna do Pará & $\mathbf{6 0}$ & Santa Luzia do Pará \\
$\mathbf{9}$ & Baião & $\mathbf{3 5}$ & Irituia & $\mathbf{6 1}$ & Santa Maria do Pará \\
$\mathbf{1 0}$ & Barcarena & $\mathbf{3 6}$ & Limoeiro Ajuru & $\mathbf{6 2}$ & Santarém Novo \\
$\mathbf{1 1}$ & Belém & $\mathbf{3 7}$ & Mãe do Rio & $\mathbf{6 3}$ & S Antônio do Tauá \\
$\mathbf{1 2}$ & Benevides & $\mathbf{3 8}$ & Mag Barata & $\mathbf{6 4}$ & S Caetano Odivelas \\
$\mathbf{1 3}$ & Bonito & $\mathbf{3 9}$ & Maracanã & $\mathbf{6 5}$ & S Domingos Capim \\
$\mathbf{1 4}$ & Bragança & $\mathbf{4 0}$ & Marapanim & $\mathbf{6 6}$ & São Francisco do Pará \\
$\mathbf{1 5}$ & Breves & $\mathbf{4 1}$ & Marituba & $\mathbf{6 7}$ & São João da Ponta \\
$\mathbf{1 6}$ & Bujaru & $\mathbf{4 2}$ & Melgaço & $\mathbf{6 8}$ & São João de Pirabas \\
$\mathbf{1 7}$ & Cachoeira Arari & $\mathbf{4 3}$ & Mocajuba & $\mathbf{6 9}$ & São Miguel do Guamá \\
$\mathbf{1 8}$ & Cachoeira Piriá & $\mathbf{4 4}$ & Moju & $\mathbf{7 0}$ & S. Sebastião B Vista \\
$\mathbf{1 9}$ & Cametá & $\mathbf{4 5}$ & Muaná & $\mathbf{7 1}$ & Soure \\
$\mathbf{2 0}$ & Capanema & $\mathbf{4 6}$ & Nova Timboteua & $\mathbf{7 2}$ & Tailândia \\
$\mathbf{2 1}$ & Capitão Poço & $\mathbf{4 7}$ & Oeiras do Pará & $\mathbf{7 3}$ & Terra Alta \\
$\mathbf{2 2}$ & Castanhal & $\mathbf{4 8}$ & Ourém & $\mathbf{7 4}$ & Tomé-Açu \\
$\mathbf{2 3}$ & Chaves & $\mathbf{4 9}$ & Paragominas & $\mathbf{7 5}$ & Tracuateua \\
$\mathbf{2 4}$ & Colares & $\mathbf{5 0}$ & Peixe-Boi & $\mathbf{7 6}$ & Ulianópolis \\
$\mathbf{2 5}$ & Concórdia PA & $\mathbf{5 1}$ & Ponta de Pedras & $\mathbf{7 7}$ & Vigia \\
$\mathbf{2 6}$ & Curralinho & $\mathbf{5 2}$ & Portel & $\mathbf{7 8}$ & Viseu \\
\hline
\end{tabular}

As informações agrícolas foram extraídas do acervo digital da Produção Agrícola Municipal (PAM) disponibilizada pelo Instituto Brasileiro de Geografia e Estatística (IBGE, 2015). Utilizaram-se os dados contidos na Tabela 1612 da PAM - IBGE contendo as variáveis de área plantada, área colhida, quantidade produzida e valor da produção da mandioca correspondentes ao período de 1990 a 2014. Para as análises deste trabalho, foram retirados os municípios com mais de cinco anos de falhas ou ausência de dados, tal que, do total de 78 municípios da área de estudo, restaram 64 municípios que são listados na Tabela 2 com os valores do rendimento médio (RM) da mandioca, considerando o período de 1990 a 2014. Nesta tabela os valores de RM foram colocados em ordem decrescente para mostrar os municípios mais produtivos da mandioca. 
Tabela 2 - Rendimento médio (RM) da mandioca (média 1990 a 2014). A ordem decrescente de RM destaca o ranking dos municípios mais produtivos na RRB.

\begin{tabular}{ll|ll|ll}
\hline Cod. e Nome município & RM & Cod. e Nome município & RM & Cod. e Nome município & RM \\
\hline 28 Dom Eliseu & $\mathbf{1 9}$ & 72 Tailândia & $\mathbf{1 3}$ & 53 Primavera & $\mathbf{1 1}$ \\
44 Moju & $\mathbf{1 9}$ & 35 Irituia & $\mathbf{1 3}$ & 15 Breves & $\mathbf{1 1}$ \\
7 Aurora do Pará & $\mathbf{1 7}$ & 27 Curuçá & $\mathbf{1 3}$ & 66 S Francisco Pará & $\mathbf{1 1}$ \\
34 Ipixuna do Pará & $\mathbf{1 6}$ & 69 S Miguel Guamá & $\mathbf{1 2}$ & 43 Mocajuba & $\mathbf{1 1}$ \\
21 Capitão Poço & $\mathbf{1 6}$ & 45 Muaná & $\mathbf{1 2}$ & 73 Terra Alta & $\mathbf{1 0}$ \\
22 Castanhal & $\mathbf{1 6}$ & 60 S Luzia Pará & $\mathbf{1 2}$ & 62 Santarém Novo & $\mathbf{1 0}$ \\
76 Ulianópolis & $\mathbf{1 6}$ & 29 Goianésia do Pará & $\mathbf{1 2}$ & 17 Cachoeira do Arari & $\mathbf{1 0}$ \\
61 S Maria Pará & $\mathbf{1 5}$ & 32 Igarapé-Miri & $\mathbf{1 2}$ & 5 Ponta de Pedras & $\mathbf{1 0}$ \\
25 Concórdia do Pará & $\mathbf{1 5}$ & 20 Capanema & $\mathbf{1 2}$ & 8 Bagre & $\mathbf{1 0}$ \\
37 Mãe do Rio & $\mathbf{1 5}$ & 74 Tomé-Açu & $\mathbf{1 2}$ & 64 S Caetano Odivelas & $\mathbf{1 0}$ \\
49 Paragominas & $\mathbf{1 5}$ & 78 Viseu & $\mathbf{1 2}$ & 47 Oeiras Pará & $\mathbf{1 0}$ \\
48 Ourém & $\mathbf{1 5}$ & 14 Bragança & $\mathbf{1 2}$ & 19 Cametá & $\mathbf{1 0}$ \\
2 Acará & $\mathbf{1 5}$ & 33 Inhangapi & $\mathbf{1 1}$ & 70 S Sebastião B Vista & $\mathbf{1 0}$ \\
13 Bonito & $\mathbf{1 5}$ & 16 Bujaru & $\mathbf{1 1}$ & 39 Maracanã & $\mathbf{1 0}$ \\
65 S Domingos Capim & $\mathbf{1 4}$ & 52 Portel & $\mathbf{1 1}$ & 42 Melgaço & $\mathbf{1 0}$ \\
9 Baião & $\mathbf{1 4}$ & 68 S João Pirabas & $\mathbf{1 1}$ & 55 Salinópolis & $\mathbf{1 0}$ \\
30 Gurupá & $\mathbf{1 3}$ & 38 Magalhães Barata & $\mathbf{1 1}$ & 56 Salvaterra & $\mathbf{1 0}$ \\
1 Abaetetuba & $\mathbf{1 3}$ & 40 Marapanim & $\mathbf{1 1}$ & 26 Curralinho & $\mathbf{1 0}$ \\
10 Barcarena & $\mathbf{1 3}$ & 63 S Antônio Tauá & $\mathbf{1 1}$ & 24 Colares & $\mathbf{1 0}$ \\
46 Nova Timboteua & $\mathbf{1 3}$ & 50 Peixe-Boi & $\mathbf{1 1}$ & 77 Vigia & $\mathbf{1 0}$ \\
31 Igarapé-Açu & $\mathbf{1 3}$ & 6 Augusto Corrêa & $\mathbf{1 1}$ & 36 Limoeiro do Ajuru & $\mathbf{9}$ \\
& & & & 59 Sta Isabel Pará & $\mathbf{9}$ \\
\hline
\end{tabular}

Os dados de precipitação consistem de médias mensais disponíveis numa grade sobre o Brasil com resolução de $0.5^{\circ}$ ( $55 \mathrm{~km}$ em latitude e longitude), os quais foram gerados através do sistema de análise de dados observacionais do Climate Prediction Center (CPC), pertencente à National Oceanic and Atmospheric Administration (NOAA). Estes dados são baseados nos registros históricos das estações meteorológicas da rede nacional (SILVA et al., 2007). Maiores detalhes sobre o controle de qualidade, técnica de análise objetiva e interpolação aplicados nestes dados podem ser encontrados em Chen et al. (2008).

Com a finalidade de se investigar os padrões oceânicos sobre o Oceano Pacífico e Atlântico, utilizou-se o conjunto de dados dispostos em pontos de grade de temperatura da superfície do mar (TSM) com resolução de $1^{\circ}$ ( 111 km2 em latitude e longitude), o qual foi compilado por Reynolds et al. (2002).

\section{MÉTODOS E PROCEDIMENTOS DE ANÁLISE}

Remoção das tendências nas séries temporais: Com a finalidade de se Remoção das tendências nas séries temporais: Com a finalidade de se remover a tendência tecnológica dos dados de rendimento médio da mandioca em cada município, foi calculada a linha de tendência linear dos dados no período de 
1990 a 2014 e em seguida os valores originais em cada ano são subtraídos dos respectivos valores da linha de tendência, conforme formulação descrita em Raffalovich (1994). Este procedimento também foi aplicado aos dados de precipitação anual.

Correlação de Spearman: É uma medida de correlação não-paramétrica para estimar a intensidade da relação entre variáveis ordinais, em que ao invés do valor observado usa-se a ordem das observações. O cálculo não é sensível a assimetrias na distribuição e nem à presença de outliers, não exigindo que os dados provenham de duas populações normais. Nos casos em que os dados não formam uma nuvem "bem comportada", com alguns pontos muito afastados dos restantes, ou em que parece existir uma relação crescente ou decrescente em formato de curva, o coeficiente de Spearman é mais apropriado. A expressão para calcular o coeficiente $\rho$ de Spearman é dada por:

$$
\rho=1-\frac{6 \sum_{i=1}^{n} d_{i}^{2}}{n^{3}-n}
$$

Em que $n$ é o número de pares ( $x i, y i)$ e di $=$ (postos de $x i$ dentre os valores de $x$ ) - (postos de yi dentre os valores de $y$ ). Se os postos de $x$ são exatamente iguais aos pontos de $y$, então todos os di serão zero e $\rho$ será 1 . 0 coeficiente varia entre -1 e 1 . Quanto mais próximo de 1 ou -1 maior será a associação entre as variáveis, sendo que o sinal positivo indica que as variáveis oscilam na mesma direção, e o sinal negativo indica as variáveis em sentido contrário.

De posse das séries temporais sem tendência, foram calculadas as correlações de Spearman entre o rendimento da mandioca (RM) e a precipitação anual (PREC_ANO), com o objetivo de avaliar as relações significantes entre o clima regional e o rendimento da cultura da mandioca. Os resultados são enfatizados para os municípios que apresentaram correlações estatisticamente significantes ao nível de 0,10 e 0,05.

Seleção dos eventos nos Oceanos Pacífico e Atlântico: Para a seleção dos episódios El Niño e La Niña sobre o Pacífico, foram extraídas as séries temporais de TSM de 1990 a 2014 nas caixas Niño3.4 e Niño3 considerando as médias sazonais de novembro do ano de formação do evento (ano 0 ) até fevereiro do ano seguinte (ano +1 ), em conformidade com estudos anteriores que demonstraram a ocorrência da fase madura do El Niño/La Niña durante o final do ano/início do ano posterior (TRENBERTH, 1997). Por outro lado, para a seleção das fases do Gradiente de TSM no Atlântico, foram extraídas as séries temporais de TSM de 1990 a 2014 considerando as médias sazonais de fevereiro a maio, o qual é o período de maior frequência do modo gradiente sobre o Atlântico intertropical (NOBRE e SHUKLA, 1996). Em seguida, foram calculadas as anomalias padronizadas de TSM nas áreas Niño3.4 e Niño3 do Pacífico e ATN e ATS do Atlântico usando a expressão:

$$
A p=\frac{T S M_{i}-T S M_{C}}{\sigma T S M_{C}}
$$

Sendo Ap a anomalia padronizada (em unidade de desvio padrão), TSMi o valor de TSM sazonal num determinado ano i, TSMc o valor médio climatológico e бTSMc o desvio padrão médio climatológico, com o subscrito c correspondendo a média 1990/2014. A Tabela 3 mostra os valores das Ap de TSM nas áreas Niño3.4, Niño3, ATN e ATS para os anos de 1990 a 2014. No caso do Atlântico, 
também foi calculada a diferença ATN-ATS, denominada de GRAD. Com base nos valores da Tabela 3, foram aplicados os seguintes critérios quantitativos para a seleção dos eventos do Pacífico e Atlântico:

- Os anos com a ocorrência concomitante de Ap positiva maior do que 0,5 desvio padrão no Niño3.4 e Niño3 (Pacífico central e leste) são considerados como episódios El Niño;

- Os anos com a ocorrência concomitante de Ap negativa menor do que 0,5 desvio padrão no Niño3.4 e Niño3 (Pacífico central e leste) são considerados como episódios La Niña.

- Os anos com a ocorrência concomitante de Ap negativa no ATN (bacia norte) e positiva no ATS (bacia sul) acompanhados de GRAD menor ou igual a -0,6 desvio padrão são considerados como episódios do Gradiente de TSM apontando para o Atlântico sul (GrSul);

- Os anos com a ocorrência concomitante de Ap positiva no ATN (bacia norte) e negativa no ATS (bacia sul) acompanhados de GRAD maior ou igual a 0,6 desvio padrão são considerados como episódios do Gradiente de TSM apontando para o Atlântico norte (GrNorte).

Os critérios mencionados acima permitiram a identificação objetiva de 07 episódios El Niño (anos 1992, 1995, 1998, 2003, 2005, 2007 e 2010), de 08 episódios La Niña (anos 1996, 1999, 2000, 2001, 2008, 2009, 2011, 2012), bem como de 06 eventos GrNorte (anos 1990, 1992, 1997, 2002, 2007, 2013) e de 05 eventos GrSul (anos 1994, 1995, 1999, 2003, 2009), conforme indicado na Tabela 3. 
Tabela 3 - Anomalias padronizadas Ap de TSM de FMAM nas regiões NATLA e SATLA e GRAD (diferença norte-sul) nos anos de 1990 a 2014. Unidade: desvio padrão.

\begin{tabular}{cccc|cccc}
\hline & \multicolumn{2}{c|}{ Oceano Pacífico: NDJF } & \multicolumn{4}{c}{ Oceano Atlântico: FMAM } \\
\hline 1990 & $-0,1$ & $-0,2$ & - & 0,2 & $-0,5$ & 0,7 & GrNorte \\
1991 & 0,3 & 0,0 & - & $-1,2$ & $-0,3$ & $-0,8$ & - \\
1992 & 1,6 & 1,3 & El Niño & 0,2 & $-1,9$ & 2,1 & GrNorte \\
1993 & 0,1 & 0,0 & - & $-0,6$ & $-1,3$ & 0,8 & - \\
1994 & 0,1 & 0,1 & - & $-1,7$ & 0,2 & $-1,9$ & GrSul \\
1995 & 1,0 & 0,8 & El Niño & $-0,2$ & 0,8 & $-1,0$ & GrSul \\
1996 & $-0,8$ & $-0,7$ & La Niña & 0,9 & 0,7 & 0,1 & - \\
1997 & $-0,4$ & $-0,7$ & - & 0,5 & $-2,3$ & 2,8 & GrNorte \\
1998 & 2,3 & 3,2 & El Niño & 1,4 & 1,2 & 0,2 & - \\
1999 & $-1,4$ & $-1,0$ & La Niña & $-1,2$ & 0,1 & $-1,3$ & GrSul \\
2000 & $-1,5$ & $-1,4$ & La Niña & $-0,1$ & 0,1 & $-0,2$ & - \\
2001 & $-0,7$ & $-0,5$ & La Niña & $-0,3$ & $-0,4$ & 0,1 & - \\
2002 & $-0,1$ & $-0,4$ & - & 0,3 & $-0,5$ & 0,9 & GrNorte \\
2003 & 1,2 & 1,0 & El Niño & $-0,7$ & 0,5 & $-1,2$ & GrSul \\
2004 & 0,3 & 0,4 & - & 0,3 & $-0,2$ & 0,5 & - \\
2005 & 0,6 & 0,5 & El Niño & 1,4 & 0,3 & 1,1 & - \\
2006 & $-0,6$ & $-0,4$ & - & 0,3 & 0,3 & 0,0 & - \\
2007 & 0,7 & 0,8 & El Niño & 0,4 & $-0,2$ & 0,6 & GrNorte \\
2008 & $-1,6$ & $-1,4$ & La Niña & 0,3 & 1,1 & $-0,8$ & - \\
2009 & $-0,6$ & $-0,5$ & La Niña & $-1,3$ & 1,3 & $-2,6$ & GrSul \\
2010 & 1,4 & 1,1 & El Niño & 2,7 & 1,7 & 0,9 & - \\
2011 & $-1,4$ & $-1,2$ & La Niña & 0,7 & 1,1 & $-0,4$ & - \\
2012 & $-0,9$ & $-0,7$ & La Niña & $-0,9$ & $-1,7$ & 0,7 & - \\
2013 & $-0,1$ & $-0,2$ & - & 0,9 & $-0,1$ & 1,0 & GrNorte \\
2014 & $-0,2$ & $-0,3$ & - & $-0,5$ & 0,0 & $-0,5$ & - \\
\hline
\end{tabular}

Critérios da análise de impactos: A análise de impactos será conduzida para os anos considerados como Extremos Climáticos Negativos (EC-) definidos pela ocorrência de El Niño e/ou GrNorte, bem como para os anos Extremos Climáticos Positivos (EC+) definidos pela manifestação de La Niña e/ou GrSul. A relação de anos de EC- é 1990, 1992, 1995, 1997, 1998, 2002, 2003, 2005, 2007, 2010 e 2013 (11 anos) e de EC+ é 1994, 1995, 1996, 1999, 2000, 2001, 2008, 2009, 2011, 2012 (10 anos).


climatológica do período de 1990 a 2014. Em seguida, foram calculados os valores de $\mathrm{RM}_{\mathrm{EC}-}$ e PREC_ANO $\mathrm{EC}_{-}$e também RM $\mathrm{EC}_{+}$e PREC_ANO $\mathrm{EC}_{+}$, sendo EC- e EC+ a composição de anos de ocorrência de Extremos Climáticos Negativos e Positivos, respectivamente. De posse desses cálculos, foram definidos os critérios de avaliação de impacto do clima no rendimento da lavoura temporária de mandioca nos municípios da RRB, levando em conta os valores e sinais das correlações significantes e os valores anômalos correspondentes as composições extremos climáticos, conforme detalhes da Tabela 4. 
Tabela 4 - Critérios adotados nas análises de impacto do clima no rendimento da mandioca

\begin{tabular}{|c|c|c|c|}
\hline Evento climático & $\begin{array}{l}\text { Critério correlação } \\
\text { (CORREL) }\end{array}$ & $\begin{array}{l}\text { Critério anomalia do } \\
\text { rendimento médio } \\
(\mathrm{RM})\end{array}$ & Tipo de impacto \\
\hline \multirow{2}{*}{$\begin{array}{c}\text { EC- } \\
\text { (El Niño e GrNorte) }\end{array}$} & CORREL $\geq 0,26$ & $\mathrm{RM}_{\mathrm{EC}-}<0$ & $\begin{array}{c}\text { Desfavorável } \\
\text { (negativo) }\end{array}$ \\
\hline & CORREL $\leq-0,26$ & $\mathrm{RM}_{\mathrm{EC}-}>0$ & $\begin{array}{l}\text { Favorável } \\
\text { (positivo) }\end{array}$ \\
\hline \multirow{2}{*}{$\begin{array}{c}\text { EC+ } \\
\text { (La Niña e GrSul) }\end{array}$} & CORREL $\geq 0,26$ & $\mathrm{RM}_{\mathrm{EC}+}>0$ & $\begin{array}{l}\text { Favorável } \\
\text { (positivo) }\end{array}$ \\
\hline & CORREL $\leq-0,26$ & $\mathrm{RM}_{\mathrm{EC}+}<0$ & $\begin{array}{l}\text { Desfavorável } \\
\text { (negativo) }\end{array}$ \\
\hline
\end{tabular}

Diagramas de caixa (Box-plot): Gráfico utilizado para avaliar a distribuição empírica de uma série de dados quantitativos, permitindo a identificação dos valores típicos (média e mediana), a assimetria, a dispersão e os dados discrepantes. Primeiramente, calculam-se a mediana, o quartil inferior (q1), o quartil superior (q3) e os extremos inferior e superior da série de dados. Em seguida, traçam-se dois retângulos (duas caixas): uma representa a "distância" entre a mediana e o q1 e o outra a "distância entre o q3 a mediana. A partir dos valores de q1 e q3 são desenhadas linhas verticais até os últimos valores não discrepantes, tanto abaixo quanto acima. Os valores típicos são a mediana e a média. Se as duas caixas tiverem "alturas" semelhantes ( $q 1$ - mediana = mediana - q3), a distribuição é dita simétrica. Quanto maiores as "alturas" das caixas maior a dispersão da série de dados, isto é, mais assimétrica é a distribuição.

\section{RESULTADOS E DISCUSSÃO}

\section{RELAÇões ENTRE O REgIME PLUVIOMÉtRICO E O RENDIMENTO DA MANDIOCA}

Os resultados obtidos nas correlações de Spearman entre séries temporais de RM da mandioca e a PREC_ANO são mostrados na Tabela 5. Evidencia-se um padrão heterogêneo com valores positivos e negativos se alternando, demonstrando que a influência do aumento ou a diminuição da precipitação no rendimento da mandioca não apresenta padrão único ao longo da RRB, ou seja, têm-se municípios com correlação direta (positiva) e indireta (negativa). Destacam-se os municípios que apresentaram correlações positivas (rendimento da mandioca aumenta/diminui nos anos com regime de chuva abundante/deficiente: Ulianópolis, Acará, Dom Eliseu, Viseu e Ipixuna do Pará com significância de 0,05, em seguida Paragominas e Concórdia do Pará com significância em 0,10, e também os municípios de Aurora do Pará, Capitão Poço, Mãe do Rio, Castanhal e Nova Timboteua com significância em 0,20. Inversamente, os municípios que apresentaram correlações negativas (produtividade da mandioca aumenta/diminui nos anos com pluviometria deficiente/abundante): Primavera, São Sebastião da Boa Vista, Santa Isabel do Pará, Augusto Corrêa, Bragança, Oeiras do Pará, Terra Alta e Santo Antônio do Tauá com significância de 0,05, em seguida Santa Luzia do Pará com significância em 0,10 e também os municípios de Curuçá, São Miguel do Guamá e Vigia com significância em 0,20. 
Tabela 5 - Correlações de Spearman entre o RM da mandioca e a PREC_ANO no período 1990 a 2014. Os municípios foram ordenados do maior para o menor valor da correlação.

\begin{tabular}{|c|c|c|c|c|c|c|c|}
\hline Cód. & Municípios & Correl & $\mathrm{p}$-valor & Cód. & Municípios & Correl & $\mathrm{p}$-valor \\
\hline 76 & Ulianópolis & $0,54 * *$ & 0,006 & 15 & Breves & $-0,01$ & 0,963 \\
\hline 2 & Acará & $0,50 * *$ & 0,011 & 13 & Bonito & $-0,05$ & 0,798 \\
\hline 28 & Dom Eliseu & $0,49 * *$ & 0,014 & 62 & Santarém Novo & $-0,06$ & 0,784 \\
\hline 78 & Viseu & $0,43 * *$ & 0,032 & 35 & Irituia & $-0,07$ & 0,756 \\
\hline 34 & Ipixuna do Pará & $0,41 * *$ & 0,043 & 43 & Mocajuba & $-0,10$ & 0,620 \\
\hline 49 & Paragominas & $0,38 * *$ & 0,060 & 38 & Mag Barata & $-0,11$ & 0,607 \\
\hline 25 & dia do Pará & $0,37 * *$ & 0,072 & 68 & S. João Pirabas & $-0,13$ & 0,520 \\
\hline 7 & do Pará & $0,33 *$ & 0,106 & 8 & Bagre & $-0,15$ & 0,461 \\
\hline 21 & o Poço & 0,3 & 0,128 & 20 & anema & $-0,16$ & 0,452 \\
\hline 37 & Rio & 0,2 & 0,161 & 30 & Ge & $-0,16$ & 0,441 \\
\hline 22 & Cast & $0,29 *$ & 0,162 & 36 & eiro Ajuru & $-0,16$ & 0,434 \\
\hline 46 & imboteua & $0,27 *$ & 0,191 & 51 & de Pedras & $-0,17$ & 0,413 \\
\hline 24 & Cola & 0,25 & 0,232 & 1 & etuba & $-0,18$ & 0,400 \\
\hline 52 & Portel & 0,23 & 0,267 & 40 & Marapanim & $-0,18$ & 0,400 \\
\hline 74 & Tomé-Açu & 0,22 & 0,285 & 17 & Cachoeira Arari & $-0,18$ & 0,383 \\
\hline 65 & ingos Capim & 0,22 & 0,290 & 66 & o do Pará & $-0,21$ & 0,314 \\
\hline 33 & Inhangapi & 0,16 & 0,449 & 45 & Muaná & $-0,25$ & 0,227 \\
\hline 9 & Baião & 0,15 & 0,485 & 61 & S. Maria Pará & $-0,25$ & 0,221 \\
\hline 16 & Buja & 0,14 & 0,492 & 55 & polis & $-0,26$ & 0,214 \\
\hline 64 & tano Odivelas & 0,14 & 0,497 & 27 & $\mathrm{Cu}$ & $-0,27^{*}$ & 0,192 \\
\hline 31 & é-Açu & 0,13 & 0,518 & 69 & S. M do Guamá & $-0,31 *$ & 0,128 \\
\hline 48 & Oure & 0,13 & 0,536 & 77 & Vigia & $-0,33 *$ & 0,113 \\
\hline 10 & Barc & 0,12 & 0,555 & 60 & S. Luzia do Pará & $-0,35^{*}$ & 0,088 \\
\hline 19 & Cam & 0,11 & 0,601 & 53 & Primavera & $-0,40 * *$ & 0,050 \\
\hline 50 & Peix & 0,07 & 0,729 & 70 & . B. & $-0,40 * *$ & 0,049 \\
\hline 26 & Curralinho & 0,05 & 0,828 & 59 & abel do Pará & $-0,41 * *$ & 0,044 \\
\hline 44 & Moju & 0,02 & 0,906 & 6 & Augusto Corrêa & $-0,42 * *$ & 0,039 \\
\hline \multirow[t]{4}{*}{72} & Tailândia & 0,02 & 0,906 & 14 & Bragança & $-0,43 * *$ & 0,035 \\
\hline & & & & 47 & Oeiras do Pará & $-0,44 * *$ & 0,028 \\
\hline & & & & 73 & Terra Alta & $-0,56 * *$ & 0,015 \\
\hline & & & & 63 & S. Antônio do Taı & $-0,57 * *$ & 0,013 \\
\hline
\end{tabular}

\section{IMPACTOS DOS ANOS EXTREMOS CLIMÁTICOS NO RENDIMENTO DA MANDIOCA}

A Figura 3 mostra os valores do 30 quartil (Q3), média e $1^{\circ}$ quartil (Q1) da precipitação $(\mathrm{mm})$ anual considerando a climatologia (Clim, média 1990 2014) e respectivos desvios percentuais (\%) das composições EC- e EC+. Os padrões de precipitação climatológica (Fig. 2a) mostram variabilidade espacial pronunciada ao longo da RRB, com os municípios do setor sudeste/leste apresentando magnitudes relativamente menores quando comparados aos municípios do setor norte/nordeste. Os desvios de precipitação associados aos anos climáticos EC- (Fig. 2b) revelam a predominância de valores negativos entre -5 e -20 na distribuição espacial dos valores de Q1 e da Med ao longo da região RRB, enquanto que para Q3 os desvios são mais intensos e com vários municípios apresentando desvios entre -15 e $-20 \%$. Inversamente, os desvios correspondentes aos anos EC+ (Fig. 2c) evidenciam a preponderância de valores positivos ao longo da RRB, com distribuição de percentuais entre $+5 \mathrm{e}$ +15 para Q3 e Med, enquanto que os desvios são mais presentes e mais 
intensos, entre +15 e $+20 \%$ para Q1. Portanto, tais resultados revelam que os episódios El Niño e GrNorte (La Niña e GrSul) deflagram a ocorrência de precipitação anomalamente negativa (positiva), com a distribuição de precipitação dos valores extremos do quartil superior - Q3 (quartil inferior - Q1) bem abaixo (acima) da média climatológica.

(a) PREC Clim

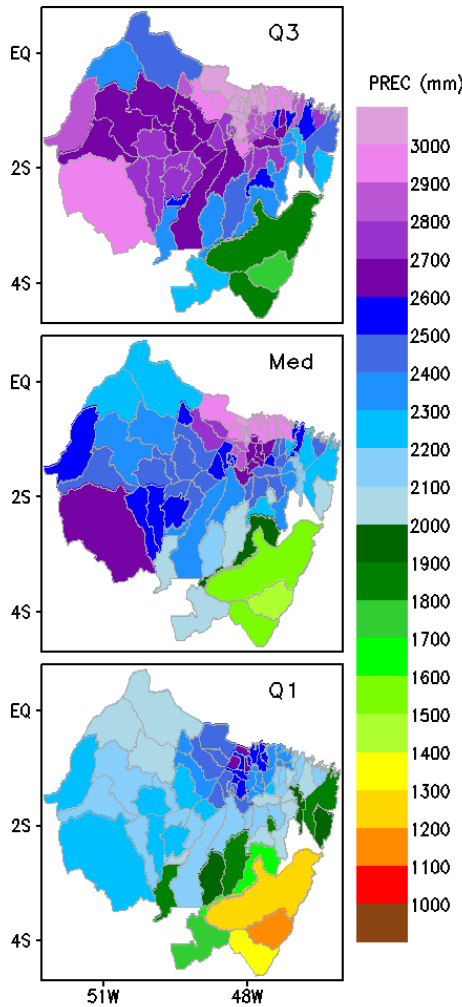

(b) \% PREC EC-

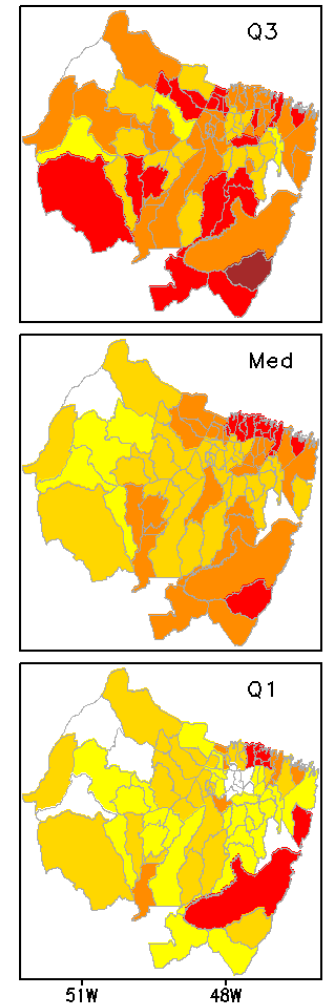

(c) \% PREC EC+
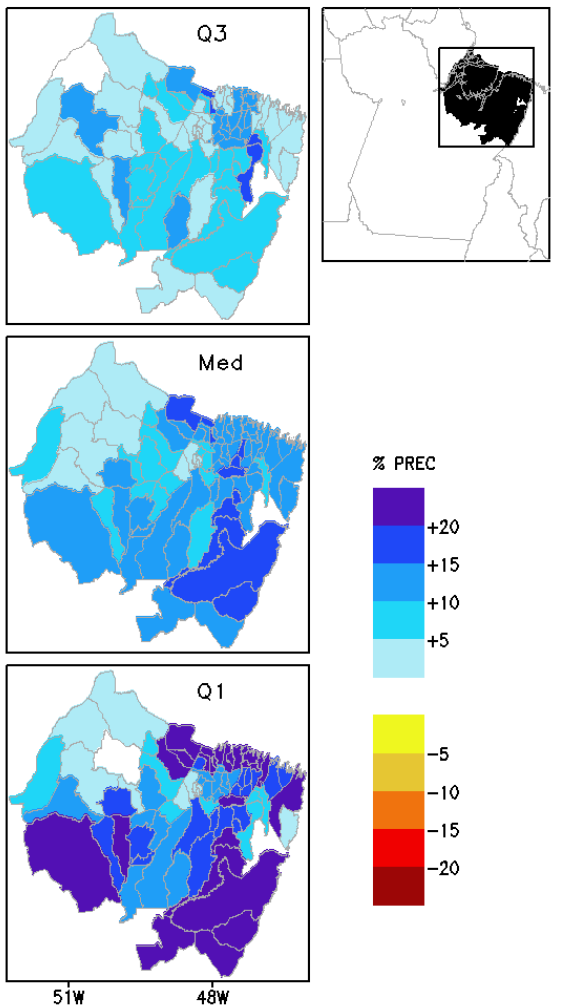

Figura 2 - Valores do $3^{\circ}$ quartil (Q3), média (Med) e $1^{\circ}$ quartil (Q1) da precipitação ( $\mathrm{mm}$ ) anual considerando: (a) climatologia (Clim, média 1990 2014) e desvios percentuais (\%) de precipitação das composições (b) EC- e (c) EC+.

Os resultados das análises de impactos são mostrados nas Figuras 3 e 4, cujos critérios (Tabela 4) levam em conta o sinal e intensidade da correlação significante, a anomalia de precipitação anual dos anos de extremos climáticos e o sinal do rendimento RM.

Os impactos dos anos climáticos EC- (predominância de chuva abaixo da média) no rendimento da mandioca são mostrados na Figura 3. Os municípios com correlação positiva apresentam condições desfavoráveis ao rendimento da mandioca, ou seja, verificam-se RM negativo (abaixo da média), com destaque para os municípios de Ulianópolis (76), Acará (2), Dom Eliseu (28), Viseu (78), Ipixuna do Pará (34), Paragominas (49), Concórdia do Pará (25), Aurora do Pará (7), Mãe do Rio (37) e Nova Timboteua (46), conforme ilustrado nos municípios em amarelo na Fig. 3. De maneira inversa, os municípios com correlação negativa exibem condições favoráveis ao aumento do rendimento da mandioca (RM positivo), principalmente em Santo Antônio do Tauá (63), Terra Alta (73), Oeiras do Pará (47), Bragança (14), Augusto Corrêa (6), Santa Isabel do Pará 
(59), São Sebastião da Boa Vista (70), Primavera (53), Santa Luzia do Pará (60), Vigia (77) e São Miguel do Guamá (69), conforme indicado nos municípios destacados em azul na Fig. 3.

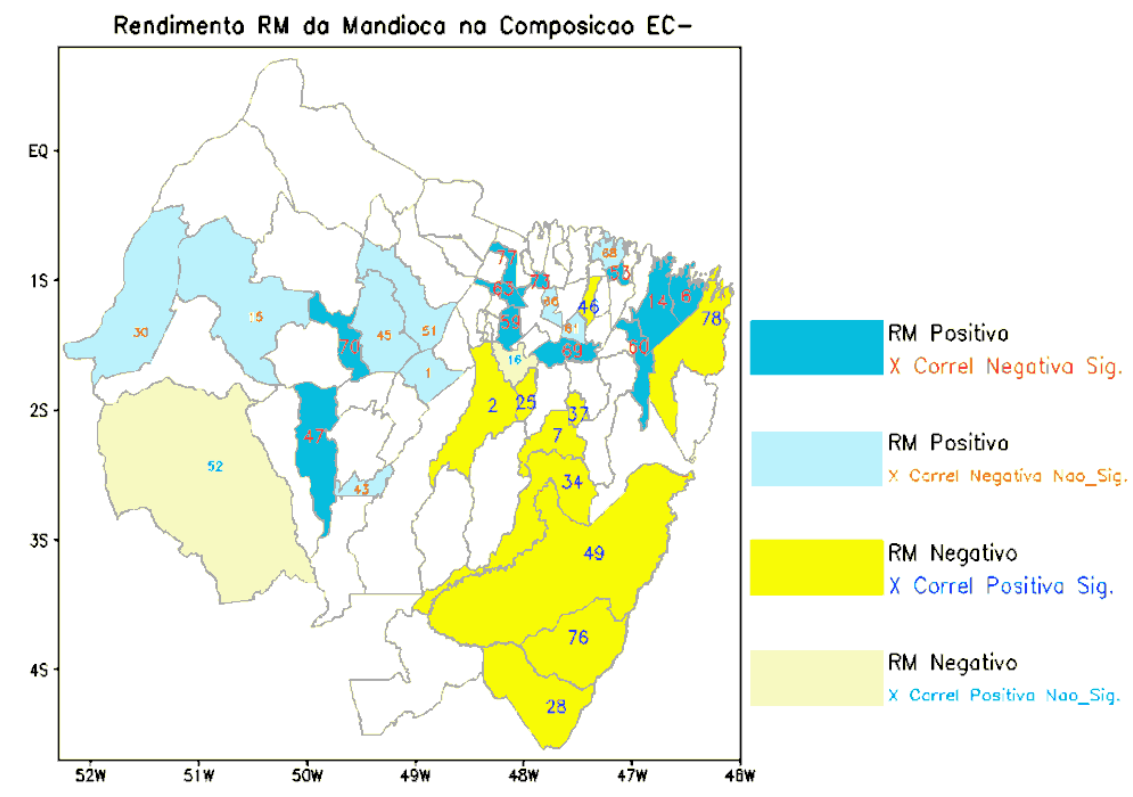

Figura 3 - Impactos dos extremos climáticos EC- no rendimento RM da mandioca. Municípios em azul indicam RM positivo (favorável ao rendimento) e em amarelo RM negativo (desfavorável ao rendimento). Números maiores e menores indicam municípios com correlação significante e não significante, respectivamente.

Os impactos dos anos climáticos EC+ (prevalência de chuva acima da média) no rendimento da mandioca são mostrados na Figura 4. Os municípios com correlação negativa apresentam condições desfavoráveis ao rendimento da mandioca, ou seja, verificam-se RM negativo, notadamente nos municípios de Santo Antônio do Tauá (63), Terra Alta (73), Oeiras do Pará (47), Bragança (14), Augusto Corrêa (6), Santa Isabel do Pará (59), São Sebastião da Boa Vista (70), Primavera (53), Santa Luzia do Pará (60), Vigia (77) e São Miguel do Guamá (69), conforme mostrado na Fig. 4 (municípios em amarelo). Por outro lado, os municípios com correlação positiva exibem condições favoráveis ao aumento do rendimento da mandioca (RM positivo), principalmente em Ulianópolis (76), Acará (2), Dom Eliseu (28), Viseu (78), Ipixuna do Pará (34), Paragominas (49), Concórdia do Pará (25), Aurora do Pará (7), Mãe do Rio (37) e Nova Timboteua (46), como ilustrado na Fig. 4 (municípios em azul). 


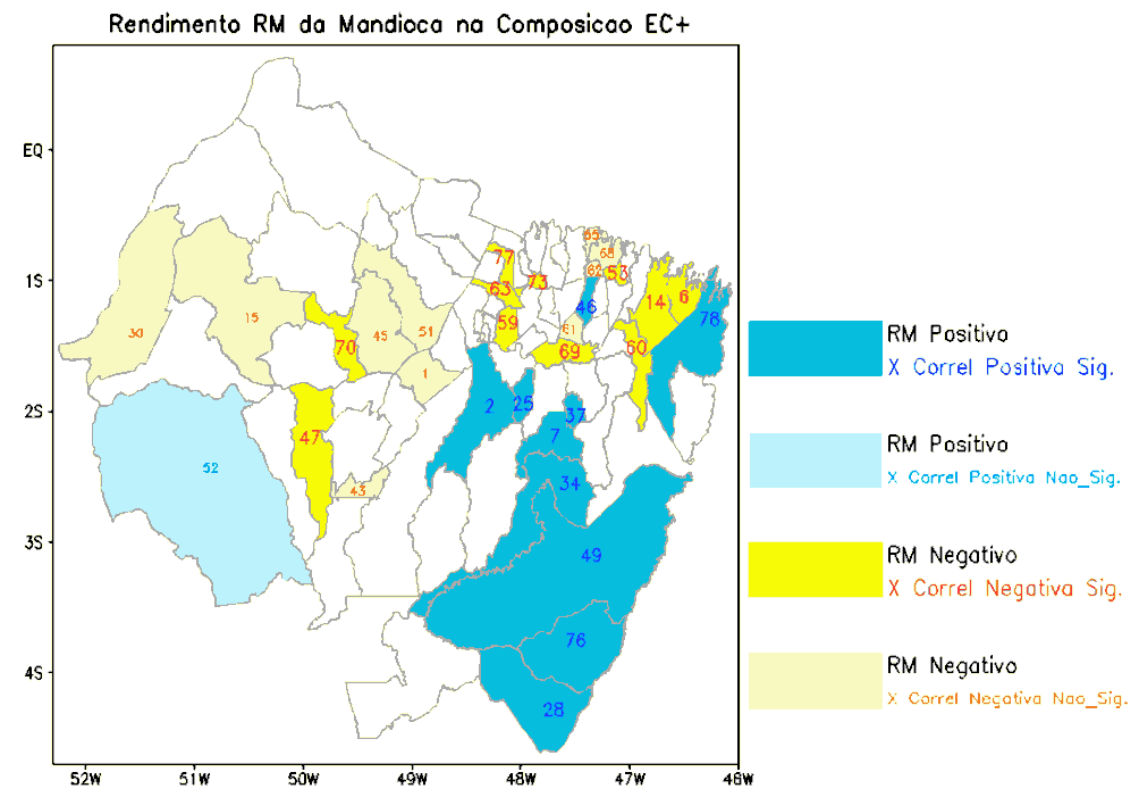

Figura 4 - Impactos dos extremos climáticos EC+ no rendimento RM da mandioca. Municípios em azul indicam RM positivo (favorável ao rendimento) e em amarelo RM negativo (desfavorável ao rendimento). Números maiores e menores indicam municípios com correlação significante e não significante, respectivamente.

Complementando as análises de impactos, foram gerados os gráficos box-plot das Figuras 5 e 6 para alguns municípios com correlação significante, visando estabelecer uma análise quantitativa do rendimento RM da mandioca dos anos climáticos extremos EC+ e EC- relativos ao padrão climatológico (média 1990_2014).

A Figura 5 mostra os impactos dos anos climáticos extremos EC+ e ECna precipitação anual (PREC_ANO) e no rendimento RM da mandioca para alguns municípios com correlação positiva significante. Os resultados demonstram que nos anos EC+ a PREC_ANO e o RM oscilam acima da média, enquanto que nos anos EC- a PREC_ANO e o RM ficam abaixo da média, notadamente nos municípios de Acará, Dom Eliseu, Viseu, Ipixuna do Pará e Paragominas.

A Figura 6 mostram os resultados em termos quantitativos dos box plots concernente aos impactos dos anos climáticos EC+ e EC- para os municípios com correlação negativa significante. Em geral, observa-se a ocorrência de PREC_ANO acima da média com RM abaixo da média nos anos EC+, enquanto que a PREC_ANO fica abaixo da média e o RM acima da média nos anos EC-, especialmente nos municípios de Ulianópolis, Terra Alta, Oeiras do Pará, Bragança, Augusto Correa e São Sebastião da Boa Vista. 

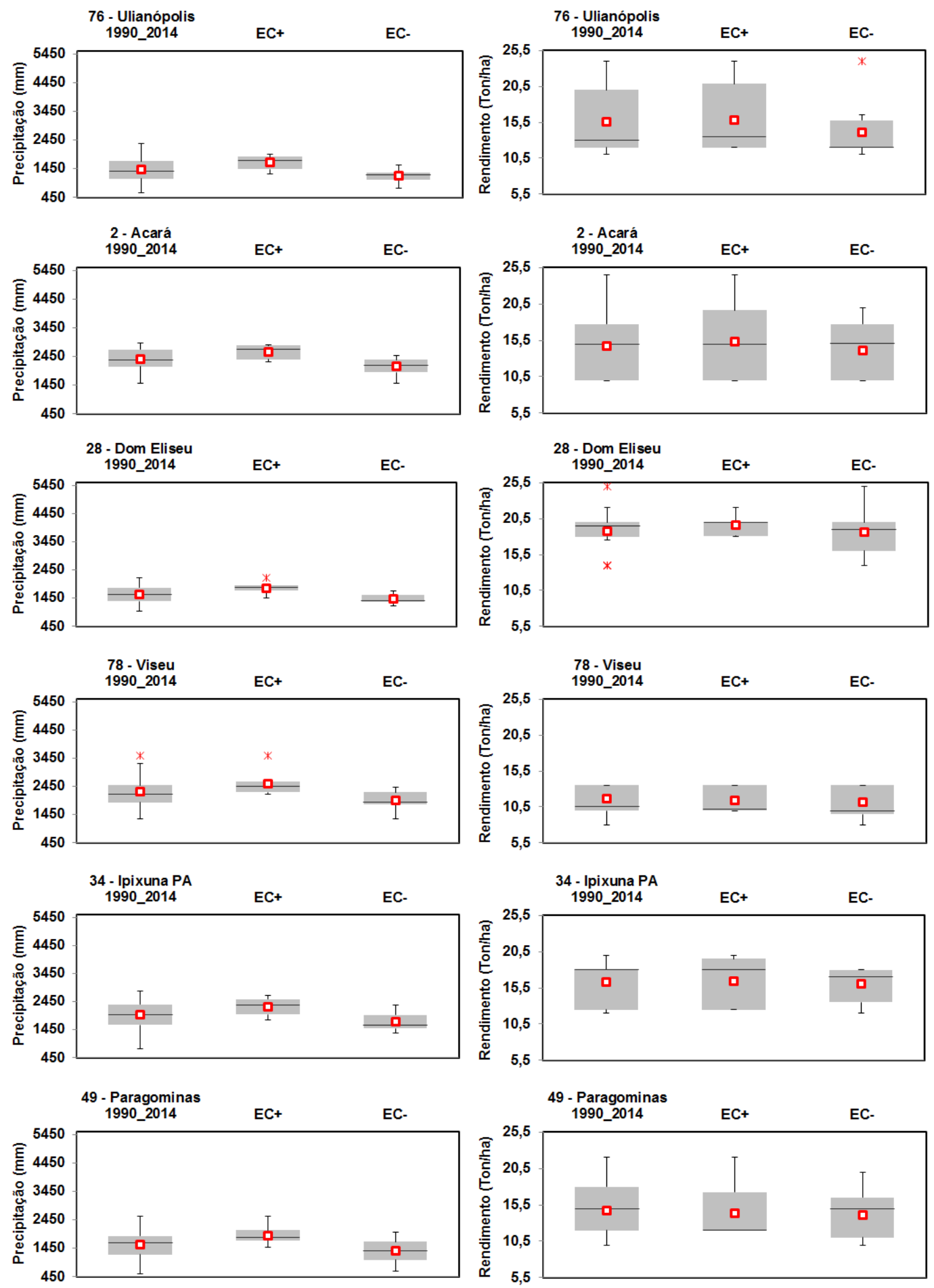

Figura 5 - Box-plot de PREC_ANO (à esquerda) e RM da mandioca (à direita) para a média 1990_2014 e composições EC+ e EC- nos municípios com correlações positivas significantes. 

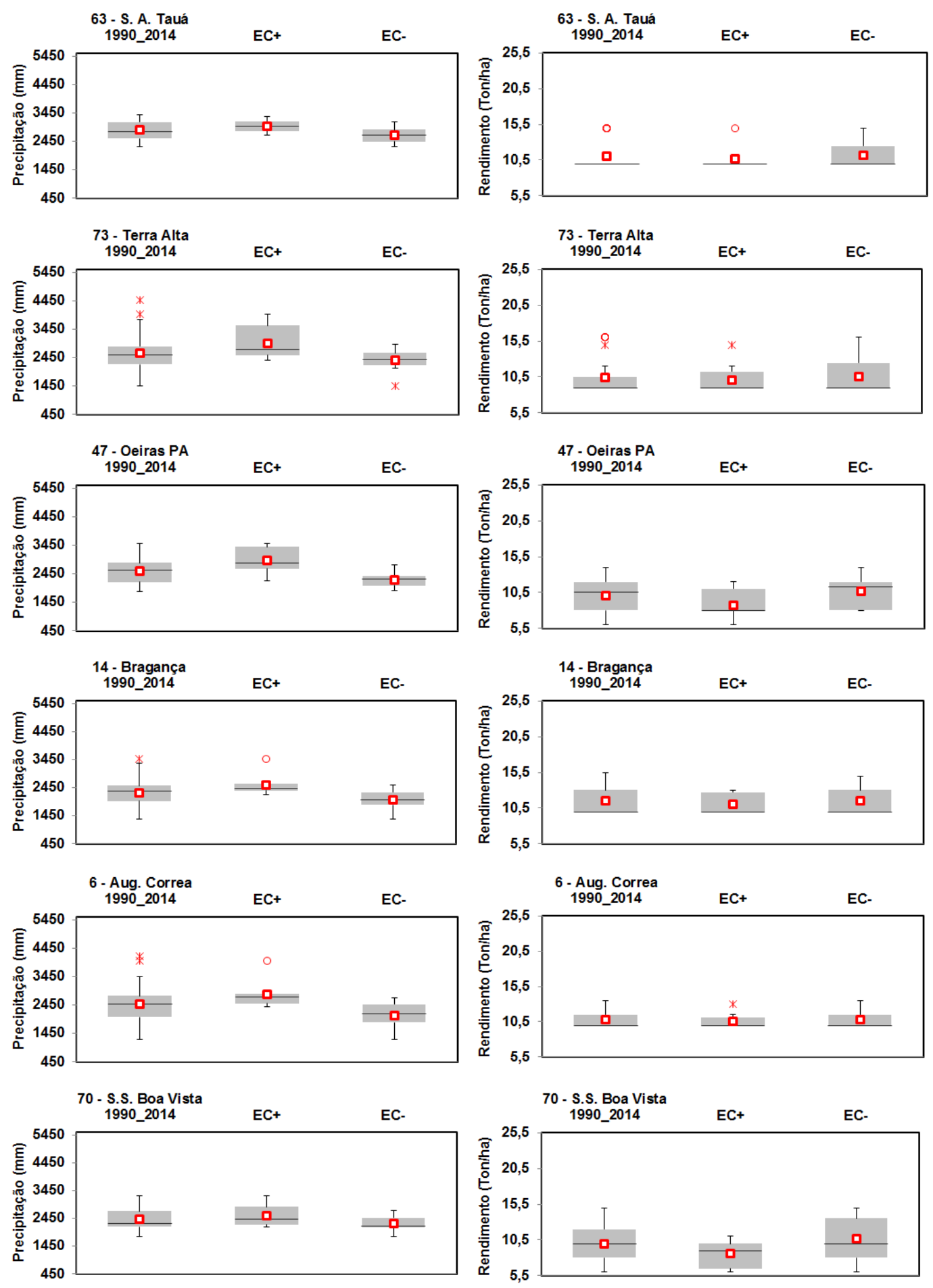

Figura 6 - Box-plot de PREC_ANO (à esquerda) e RM da mandioca (à direita) para a média 1990_2014 e composições EC+ e EC- nos municípios com correlações negativas significantes. 
Segundo Modesto Junior et al (2016), com respeito aos quatro municípios do estado do Pará que apresentam as maiores produções de mandioca, somente em Acará o preparo da área é o tradicional praticado na agricultura familiar, isto é, do tipo corte-queima que apresenta alguns inconvenientes como poluição ambiental, erosão, perda de nutrientes, além de tratar-se de um trabalho penoso com grande desgaste físico do agricultor. Esse sistema só permite bom rendimento no primeiro ano, pois no segundo a produtividade das culturas diminui, aumenta a infestação de ervas daninhas, e o número de capinas. Com isso, o agricultor abandona a área, deixando-a em pousio, derrubando nova capoeira para continuar a produzir alimentos. Os outros três municípios, Ipixuna do Pará, Bragança e Aurora do Pará, o preparo da área é semi ou totalmente mecanizado. Além disso, a variedade que é plantada em Acará é a do tipo Crioula, enquanto que nos outros três municípios a variedade é do tipo BRS Poti/BRS Mari. Nos municípios com as menores produções, como Limoeiro do Ajuru, Ponta de Pedras, Salvaterra, Bagre, Curralinho, Santa Isabel do Pará e Capitão Poço, o preparo da área é de corte e queima e a variedade é Crioula, com exceção de Capitão Poço, cujo sistema é de plantio é semi ou totalmente mecanizado. Dom Eliseu e Moju são os municípios que apresentam os maiores rendimentos médios. O sistema de plantio é de corte e queima e a variedade é do tipo Crioula. O terceiro (Aurora do Pará) e o quarto (Ipixuna do Pará) com maiores produtividade praticam o sistema de plantio semi ou totalmente mecanizado e a variedade plantada é a BRS Poti/Mari. Em todos os municípios, o plantio ocorre em dezembro e Junho, com exceção de Dom Eliseu que ocorre em outubro. O solo em todos os municípios é do tipo Latossolo Amarelo (EMBRAPA, 2016).

A produtividade está condicionada a forma e manejo dos agricultores e relacionando com trabalhos realizados no Estado do Pará, como Modesto Júnior et al. (2011) que alcançaram produtividade média de $25.560 \mathrm{~kg}$ ha-1, utilizando práticas comumente relatadas na agricultura familiar, como cultivos em leira para diminuir a perda de raiz por encharcamento. Manejar o solo é, portanto, utilizá-lo adequadamente, tendo como base a relação dos vários fatores que afetam a produtividade agrícola, tais como: a rotação de culturas, o uso de adubos verdes, a fertilização, a irrigação correta e o cultivo adequado. Já a conservação do solo é a designação coletiva dos programas de prevenção e controle à erosão, da excessiva perda de nutrientes e, de maneira geral, da perda de sua capacidade de sustentar a vegetação natural e/ou a agricultura (ALVES, MODESTO JÚNIOR, ANDRADE, 2008; MODESTO JUNIOR, ALVES, SILVA, 2011).

A mandioca se apresenta como opção para garantir a qualidade de vida das comunidades tradicionais na Amazônia, além de reduzir o fluxo migratório do homem do campo para os grandes centros urbanos. A produção de mandioca ao longo do tempo tem alçando a adoção de novas tecnologias tanto de pesquisas como de manejo, o cultivo racional tem sido importante para a exploração da cultura, haja vista sua contribuição na oferta em função da grande demanda de mercado. No Pará, embora o sistema de cultivo da mandioca ser praticado por pequenos produtores e apresentar ausência de mecanização, mesmo assim a capacidade de gerar lucro com níveis significantes de produção é alta (RICHETTI, 2007; BALSAN, 2006).

Apesar das condições edafoclimáticas favoráveis, o sistema de produção na região norte do Brasil é marcado por adoção de práticas rústicas de plantio, 
visto que se observa grande parte dos camponeses utilizando na cadeia produtiva da mandioca, o cultivo vinculado ao sistema de derruba, queima e coivara (ALVES et al., 2008) e adoção de ferramentas estritamente manuais, que para Mazoyer e Roudart (2010), ratifica uma completa ausência da mecanização na lavoura, práticas essas que impactam de forma direta e negativamente a produção. E por outro lado, forçam os produtores a buscarem novas áreas de cultivos, expandindo a fronteira agrícola na região (BUAINAIN, 2007).

Resultados dessas práticas de cultivo resultam em baixa produtividade, pois segundo levantamento do Instituto Brasileiro de Geografia e Estatística (IBGE, 2010), os municípios do estado do Pará apresentam valores baixos de rendimento médio de raízes de mandioca, sendo o volume produzido da ordem de $14,68 \mathrm{t} / \mathrm{ha}$, valor bem inferior às possibilidades da região, que segundo Alves et al. (2008) a produtividade está muito abaixo das que poderiam ser obtidas. Porém este problema pode ser suprimido a partir da adoção de tecnologias e adequações técnicas, dentre elas, a denominada "trio da produtividade" o que pode duplicar o rendimento, chegando na ordem de 27,64 t/ha.

\section{CONCLUSÃO}

As análises da distribuição espacial das correlações de Spearman entre rendimento médio (RM) de mandioca e a precipitação pluviométrica nos municípios da Região Rural da Metrópole de Belém (RRB) no estado Pará no período de 1990 a 2014, de uma maneira geral, mostraram um padrão heterogêneo com valores positivos e negativos se alternando ao longo da região. Assim, a influência do aumento ou diminuição no total de precipitação (tanto no período anual quanto nos períodos mais e menos chuvoso) no RM da mandioca não apresenta padrão único ao longo da RRB, ou seja, se observam municípios com correlações direta e indireta. A correlação entre o RM de mandioca e o total anual de chuva mostrou que os municípios de Ulianópolis, Acará, Dom Eliseu, Ipixuna do Pará e Viseu apresentaram correlações positivas com nível de significância de 0,05, ou seja, o RM de mandioca aumenta (diminui) nos anos com o total anual acima (abaixo) da Normal Climatológica. Por outro lado, os municípios, cuja correlação foi indireta, isto é, negativa, em que o RM de mandioca aumenta (diminui) nos anos com o total anual abaixo (acima) da Normal Climatológica, com nível de significância de 0,05, foram Santo Antônio do Tauá, Terra Alta, Oeiras do Pará, Bragança, Augusto Correa, Santa Isabel do Pará, São Sebastião da Boa Vista e Primavera. Os impactos no RM de mandioca decorrentes dos anos climáticos extremos EC- (El Niño e Gradiente Norte) e EC+ (La Niña e Gradiente Sul) mostraram também um padrão heterogêneo, não afetando a região como um todo. Observou-se, de maneira geral, que o impacto em alguns municípios foi favorável e em outro desfavorável ao RM de mandioca.

\section{REFERÊNCIAS BIBLIOGRÁFICAS}

ALVES, R. N. B.; MODESTO JúNIOR, M. S.; ANDRADE, A. C. S. O trio da produtividade na cultura da mandioca: estudo de caso de adoção de tecnologias na região no Baixo Tocantins, Estado do Pará. In: Congresso da Associação 
Brasileira das Instituições de Pesquisa Tecnológica, 2008, Campina Grande. Brasília, DF: ABIPTI, 2008.

ARRUDA, H.V.; PINTO, H. A simplified gamma probability model for analysis of the frequency distribution of rainfall in the region of Campinas, SP - Brazil. Agricultural and Forest Meteorology, v. 22, n. 2, p. 101-108. 1980.

AYOADE, J. O. Introdução à climatologia para os trópicos. São Paulo: Difel, 1986.

BALSAN, R. Impactos decorrentes da Modernização da agricultura brasileira. CAMPO-TERRITÓRIO: Revista de Geografia Agrária, v. 1, n. 2, 2006.

BUAINAIN. A, M. Agricultura familiar e inovação tecnológica no Brasil: características, desafios e obstáculos. Campinas, SP: Ed. Unicamp, 2007.

CAMARGO FILHO, W.P.; ALVES, H.S. Produção e mercado de mandioca: análise de preços ao produtor. Informações Econômicas, v. 34, n. 9, 2004.

CHEN, M.; SHI, W.; XIE, P. Assessing objective techniques for gauge-based analyses of global daily precipitation. Journal of Geophysical Research, v. 113, p. D04110, 2008.

DIAS, C. A. C.; MARTINEZ, A. A. Mandioca: Informações importantes. Campinas: Coordenadoria de Assistência Técnica Integral, n. 190, p. 20, 1986.

EMBRAPA. Mapas de Solos e de Aptidão Agrícola das Áreas Alteradas do Pará. Embrapa Amazônia Oriental. Embrapa Solos. Ministério da Agricultura, Pecuária e Abastecimento - MAPA, 2016.

FERREIRA, D. B. Relações entre a variabilidade da precipitação e a produtividade agrícola de soja e milho nas regiões sul e sudeste do Brasil. São José dos Campos: (INPE-13599-TDI/1038), p. 123, 2005.

FOOD AND AGRICULTURE ORGANIZATION OF THE UNITED NATIONS (FAO). Dados da produção mundial da mandioca. Disponível em: <http://faostat.fao.org/site/339/default.aspx>. Acesso em: 17 nov. 2014.

HAESBAERT, R. Região, diversidade territorial e globalização. Geographia, v. 1, n. 1 , p. 15-39, 1999.

INSTITUTO BRASILEIRO DE GEOGRAFIA E ESTATÍSTICA - IBGE. Estados. 2010. Disponível em:<http://www.ibge.gov.br/estadosat/perfil.php?sigla=pa\%3E>. Acesso em: 12 de mar. 2016.

INSTITUTO BRASILEIRO DE GEOGRAFIA E ESTATÍSTICA - IBGE. Sistema de Recuperação Automática (SIDRA). Pesquisa Agrícola Municipal. Disponível em: <www.sidra.ibge.gov.br>. Acesso em: mar. 2015.

MANOSSO, F. C. A produtividade de soja, trigo e milho e suas relações com a precipitação pluviométrica no município de Apucarana-PR no período de 1968 a 2002. Geografia, v. 14, p. 87-98, 2005.

MAYOZER, M.; ROUDART, L. História das agriculturas no mundo: do neolítico à crise contemporânea. São Paulo. Ed. UNESP; Brasília-DF: NEAD. 2010. 568 p.

MODESTO JÚNIOR, M. S.; ALVES, R. N. B. Cultura da mandioca: aspectos socioeconômicos, melhoramento genético, sistemas de cultivo, manejo de 
pragas e doenças e agroindústria. 2016. EMBRAPA, Brasília, DF. Disponível em: https://www.embrapa.br/amazonia-oriental/publicacoes.

MODESTO JÚNIOR, M. S.; ALVES, R.N. B.; SILVA, E. S. A. Produtividade de mandioca cultivada por agricultores familiares na região do Lagos, município de Tracuateua, estado do Pará. Amazônia: Ciência \& Desenvolvimento, v. 6, n. 12, 2011.

NOBRE, P.; SHUKLA J. Variations of SST, wind stress and rainfall over the tropical Atlantic and South America. Journal of Climate, v.9, p. 2464-2479, 1996.

NORMANHA, E. S. Clima e terra para cultura da mandioca. O Agronômico, Campinas, v. 1, p.77-79, 1941.

RAFFALOVICH, L. E. Detrending Time Series: A Cautionary Note. Sociological Methods \& Research, v. 22, n. 4, p. 492-519, 1994.

REYNOLDS, R. W.; RAYNER, N. A.; SMITH, T. M.; STOKES, D. C.; WANG, W. An improved in situ and satellite SST analysis for climate. Journal of Climate, v. 15, p. 1609-1625, 2002.

RICHETTI, A. Custo de produção de mandioca industrial, safra 2007. Revista Raízes e Amidos Tropicais, v. 3, 2007.

SILVA, V. B. S.; KOUSKY, V. E.; SHI, W.; HIGGINS, R. W. An improved gridded historical daily precipitation analysis for Brazil. Journal of Hydrometeorology, $v$. 8, p. 847-861, 2007.

TERACINES, E. B. Impactos econômicos do El Niño 97/98 na produção agrícola brasileira. In: Simpósio Brasileiro De Climatologia Geográfica, 2000, Rio de Janeiro-RJ. Clima e Ambiente (Sustentabilidade, Riscos e Impactos), 2000 .

TRENBERTH, K. E. The definition of El Niño. Bull. Amer. Meteor. Soc., v. 78, p.2771-2777, 1997.

VELTZ, P. Zonas, polos, redes: la economia de archipiélago. In: Mundialización, Ciudades y Territorios - La Economía de Archipiélago. Barcelona: Ariel, 1999, p. 53-65.

VILPOUX, O. A cadeia de mandioca no Brasil. Campo Grande: SEBRAE-MS, 2006, p. 82 